derivatives of steroid hormones and vitamin D3 synthesis. We also identified mevalonate and 7-dehydrodemosterol, the intermediates of cholesterol biosynthesis. The concentrations of FFA biosynthesis intermediates were higher with LPON compared with oleate (3-oxotetradecanoate $(p=0.005)$ and 3-oxo-hexadecanoate $(p=0.02))$. Although mevalonate $(p=0.37)$ and 7 -dehydrodesmosterol $(p=0.46)$ levels were higher with oleate than that seen with LPON, these differences did not reach statistical significance. In contrast, bile acids were significantly elevated with oleate than LPON ((taurocholate $(p=0.002)$, glycocholate $(p=0.001), \quad(6 \mathrm{RS})-22$ oxo-23,24,25,26,27-pentanorvitamin D3 6,19-sulphur dioxide adduct $(p=0.04)$ and 1,25-dihydroxy-2,4-dinor-1,3-secovitamin D3 $(p=0.0006)$.

Conclusion These data suggest that, aside from enhanced ketogenesis, impaired mitochondrial function is also associated with acetyl-coA diversion towards FFA synthesis, but not mevalonate pathways. These differences are likely to reflect cellular demand in the presence of decreased ATP formation with mitochondrial dysfunction.

Competing interests None declared.

\section{PM0-131 FGF INDUCIBLE PROTEIN 14 IS UPREGULATED IN NEOVESSELS DURING CHRONIC INFLAMMATORY LIVER DISEASE AND PROMOTES INTRAHEPATIC ENDOTHELIAL CELL ANGIOGENESIS IN VITRO FOLLOWING STIMULATION VIA TWEAK}

doi:10.1136/gutjnl-2012-302514b.131

${ }^{1} \mathrm{M}$ Munir, ${ }^{1} \mathrm{E}$ Humphreys, ${ }^{1} \mathrm{~A}$ Wilhelm, ${ }^{1} \mathrm{~B}$ Stephenson, ${ }^{1} \mathrm{~J}$ Caamano, ${ }^{2} \mathrm{~L}$ Burkly, ${ }^{1} \mathrm{D}$ Adams, ${ }^{1} \mathrm{~S}$ Afford. ${ }^{1}$ Centre for Liver research, University of Birmingham, Birmingham, UK; ${ }^{2}$ Biomedical Research Institute, Biogenldec, Boston, USA

Introduction TWEAK and Fn14 members of the TNF superfamily of ligands and receptors collectively regulate a diverse range of immune, inflammatory and regenerative responses. Recent studies indicate a potential role of TWEAK and Fn14 in tissue repair following liver injury where they may promote angiogenesis and neovessel growth. TWEAK/Fn14 could therefore facilitate inflammatory cell recruitment and promote portal associated lymphoid tissue development during inflammation.

Aims (1). To investigate TWEAK/Fn14 expression in human liver tissue during chronic liver disease. (2) To study Fn14 expression in isolated intra-hepatic endothelial cells (IHEC). (3) To determine angiogenic responses of IHEC to TWEAK.

Methods Tissue sections from explanted human livers at the time of hepatobiliary surgery, including normal donor; normal tissue adjacent to malignant lesions; HCV; ALD; NASH; Chronic Allograft Rejection; PSC and PBC, were subjected to immunohistochemistry or dual immunofluorescence using antibodies to TWEAK, Fn14 and phenotypic markers CD31 and CD68. Isolated IHEC were cultured with combinations of TNF $\alpha$, IFNg, FGF, and assessed for TWEAK/ Fn14 expression using flow cytometry. In addition IHEC were incubated with recombinant TWEAK in presence \pm TNF $\alpha$ and assessed using a matrigel angiogenesis assay for vessel formation and branching.

Results Fn14 expression was low in normal tissue in portal vessels and sinusoids, whereas in disease portal neovessels (CD31 +ve) were highly positive for Fn14. TWEAK expression was low in normal tissue but highly expressed in CD68+ve monocytic cells surrounding areas of neovascularisation and inflammatory cell aggregation. Fn14 expression significantly up-regulated on isolated IHEC when stimulated with the TNF $\alpha$. Confocal imaging showed that the expression of Fn14 was predominantly cytoplasmic unless stimulated with TNF which enhanced cell surface expression. IHEC were consistently negative for TWEAK and TWEAK stimulation increased IHEC angiogenesis, where a change in cell morphology and enhanced branching of capillary like structures was observed. Pre-incubation with Fn14 antagonistic mAb completely abrogated vessel formation and branching.

Conclusion These new data show that Fn14 activation stimulates neovessel branching in IHEC and that TNF- $\alpha$ promotes mobilisation of cytoplasmic Fn14 to the cell surface suggesting an important regulatory role for TWEAK/Fn14 in neovascularisation and portal lymphoid aggregation during hepatic inflammation.

Competing interests None declared.

\section{PM0-132 HISTONE VARIANT MACROH2A1 KNOCK-OUT WORSENS HIGH FAT DIET-INDUCED SYSTEMIC INSULIN RESISTANCE, GLUCOSE INTOLERANCE AND HEPATIC STEATOSIS IN MICE}

doi:10.1136/gutjnl-2012-302514b.132

M Vinciguerra, ${ }^{*}$ C Podrini, A Greco. Institute of Hepatology, Foundation for Liver Research, London, UK

Introduction Hepatic steatosis is a major risk factor for the development of severe liver damage, including fibrosis, cirrhosis and hepatocellular carcinoma. It often exists as a co-morbidity factor with diabetes type I/II and with other manifestations of the metabolic syndrome. Recent studies highlight the importance of an epigenetic basis for the development of steatosis based on macroH2A1. MacroH2A1 is a histone variant of histone H2A, which possesses an additional protein domain called macro. When incorporated into the chromatin of hepatocytes, macroH2A1 regulates gene expression. Two alternatively spliced isoforms of macroH2A1 exist, which have been shown to be markers of breast, skin and lung cancer. Whole-body knock out of macroH2A1 in mice induces glucose intolerance and changes in genes regulating hepatic lipid metabolism. However, overt hepatic steatosis was not observed and the significance of these findings is unclear. We hypothesised that macroH2A1 could be involved in the pathogenesis of hepatic steatosis induced in the high fat-diet mouse model of metabolic syndrome.

Methods Wild type and homozygous knock-out male mice were placed on a high fat diet (Western diet, $42 \%$ of energy intake from saturated fats) for 8 weeks. At the end of the treatment, measurement of insulin sensitivity and glucose tolerance (ITT and GTT) were performed. Mice were sacrificed, and plasma and liver tissue harvested for further assays, including protein, gene expression and immunohistochemistry analyses.

Results Deterioration in insulin resistance and glucose intolerance induced by high fat diet was observed in macroH2A1 knock-out mice, compared to wild type littermates. Moreover, lack of macroH2A1 in mice significantly worsened the increase in circulating non-esterified fatty acids (NEFA) and the hepatic intracellular content of tryglicerides induced by high fat diet. Gene expression studies unveiled an increase in the hepatic expression of lipoprotein lipase (Lpl) and fatty acid transporter CD36 in knock-out mice.

Conclusion MacroH2A1 is a regulator of hepatic fat accumulation and its absence worsens hepatic steatosis and systemic imbalances upon a high fat regimen in mice. This could be due to a direct effect of macroH2A1 on chromatin structure and on the expression of key genes involved in liver lipid metabolism.

Competing interests None declared.

\section{REFERENCES}

1. Changolkar LN, Costanzi C, Leu NA, et al. Developmental changes in histone macroH2A1-mediated gene regulation. Mol Cell Biol 2007;27:2758-64.

2. Boulard M, Storck S, Cong R, et al. Histone variant macroH2A1 deletion in mice causes female-specific steatosis. Epigenetics Chromatin. 2010;3:8. 